\title{
Ritmo circadiano da taxa respiratória de Tetragonisca angustula fiebrigi (Schwarz), T. a. angustula (Latreille) e Trigona spinipes (Fabricius) (Hymenoptera, Apidae, Meliponinae)
}

\author{
Edson Aparecido Proni \& Oilton José Dias Macieira \\ Departamento de Biologia Animal e Vegetal, Universidade Estadual de Londrina. Campus Universitário, Caixa Postal 6001, \\ 86051-900 Londrina, Paraná, Brasil. E-mail: proni@uel.br; macieira@uel.br
}

\begin{abstract}
Circadian rhythm of the respiratory rate of Tetragonisca angustula fiebrigi (Schwarz), T. a. angustula (Latreille) and Trigona spinipes (Fabricius) (Hymenoptera, Apidae, Meliponinae). The circadian rhythm of the mean respiratory rates $\left(\mathrm{VO}_{2}\right)$ was determined on isolated workers of Tetragonisca angustula fiebrigi (Schwarz, 1938), T. a. angustula (Latreille, 1807) and Trigona spinipes (Fabricius, 1793) in normal cycle (day/light and night/dark) conditions, during springtime. Oxygen consumption was determined at $30^{\circ} \mathrm{C}$ by using a Warburg respirometer. The statistical analysis of the results revealed significant interactions among time intervals and between the species. Breathing rates were compared using the Tukey's test. A clear circadian pattern of the metabolic rhythm was observed for both species. It showed a larger consumption of oxygen during the day, with a peak between 10:00 and 15:00. At night the consumption decreased, reaching the minimum levels between 2:00 and 3:00. Tetragonisca angustula angustula and $T$. a. fiebrigi did not show significant differences between them but they did differed from $T$. spinipes, which showed larger respiratory rates.

KEY WORDS. Abelhas sem ferrão, consumo de oxigênio, metabolismo respiratório.
\end{abstract}

RESUMO. Foi determinado em operárias isoladas de Tetragonisca angustula fiebrigi (Schwarz, 1938), T. a. angustula (Latreille, 1807) e Trigona spinipes (Fabricius, 1793) o ritmo circadiano das taxas respiratórias médias $\left(\mathrm{VO}_{2}\right)$ em condições de ciclo normal (dia/ claro e noite/ escuro), durante a primavera. $\mathrm{O}$ consumo de oxigênio foi determinado a $30^{\circ} \mathrm{C}$ usando um respirômetro de Warburg. A análise estatística dos resultados revelou interações significativas entre intervalos de tempo e entre as espécies. Foram comparadas as taxas respiratórias usando o teste do Tukey. Um padrão definido de ritmo circadiano metabólico foi observado para ambas as espécies, apresentando um maior consumo de oxigênio durante o dia, com um pico entre 10:00 e 15:00. À noite o consumo diminuiu, alcançando os níveis mínimos entre 2:00 e 3:00. Tetragonisca angustula angustula e $T$. a. fiebrigi não apresentaram diferenças significativas entre si, mas diferiram de $T$. spinipes, que apresentou taxas respiratórias maiores.

PALAVRAS CHAVE. Oxygen consumption, respiratory metabolism, stingless bees.

Os meliponíneos são encontrados em regiões tropicais e subtropicais, até $30^{\circ}$ de latitude norte e sul. Estes insetos têm a característica peculiar de apresentarem o ferrão atrofiado, por isso são conhecidas como abelhas sem ferrão ou "stingless bees". Segundo Nogueira-Neto (1970) as abelhas Tetragonisca angustula fiebrigi (Schwarz, 1938) e Tetraginisca angustula angustula (Latreille, 1807) vulgarmente conhecidas como "jataí", são encontradas desde o México até o Rio Grande do Sul. Por sua vez as abelhas Trigona spinipes (Fabricius, 1793), também conhecidas como "irapuá", ocorrem desde o Ceará até o Rio Grande do Sul, no Paraguai e na Província de Missões (Argentina). As abelhas são consideradas de vital importância para os ecossistemas, devido à eficiência como agentes polinizadores.
Segundo Roubik (1979), a eficácia no ciclo reprodutivo da maioria de espécies vegetais nativas das regiões tropicais, tem como motivo a grande variabilidade no tamanho dos indivíduos das diferentes espécies dessas abelhas. O processo de polinização realizado pelas abelhas diminui o isolamento reprodutivo vegetal, resultando num aumento da biodiversidade (MiCHENER 1974, Price 1975, Bawa \& Opler 1975, Jansen 1975, RoubiK 1979, Aвsy et al. 1984).

De acordo com KeRR et al. (1996), nas florestas brasileiras as abelhas indígenas constituem-se nos principais agentes polinizadores de $40 \%$ a $90 \%$ das espécies vegetais, enquanto outros animais como aves, borboletas e alguns mamíferos desempenham o papel polinizador restante. Estudos referentes à 
associação inseto-planta, especificamente entre meliponíneos e vegetais nativos da região de Manaus-AM, verificaram que a extinção de espécies nativas de abelhas acelera o processo de extinção de espécies vegetais desequilibrando os ecossistemas (Absy \& Kerr 1977, Kerr et al. 1978, Absy et al. 1980). Assim sendo, toda e qualquer possibilidade de desenvolver estudos destinados a conhecer a biologia básica dos meliponíneos, poderá trazer soluções práticas na conservação e manejo dos ecossistemas atuais (KerR et al. 1996).

Estudos sobre ritmo circadiano e atividade de insetos, de um modo geral, foram realizados por TwEEDY \& STEPHEN (1971), Kapoor (1972), Chiba et al. (1973), BANKS et al. (1975), TAKAHASHIdel-Bianco \& Hebling (1992), Moore \& Rankin (1993), Frisch \& Koeniger (1994), Moritz \& Kryger (1994), Moore et al. (1998), Bellusci \& Marques (2001), Moore (2001), Proni et al. (2001) e BLOCH et al. (2002).

Segundo Harker (1958), a característica de maior interesse no estudo de ritmo de 24 horas, não seria o fato de certas atividades ocorrerem todas neste intervalo, mas sim que tais repetições persistissem na ausência de mudanças ambientais normais. Dessa maneira podem ser observados dois tipos de ritmos: A) aqueles que ocorrem como resposta imediata para mudanças no ambiente (ritmos exógenos); B) os que persistem quando as condições no ambiente são mantidas constantes (ritmos endógenos).

Os ritmos biológicos, segundo CipolLA-Neto et al. (1988), podem ser estudados através da cronobiologia, que pode ser entendida como o estudo da organização temporal da matéria viva. Assim, o ritmo biológico, que está presente em todos seres vivos (devido ao seu caráter ubíquo), parece ter-se originado desde os primórdios da vida, tendo contribuído como fator organizador da matéria viva. Portanto, a ritmicidade encontrada em animais e plantas não é apenas expressão reflexa dos eventos temporais do meio externo, mas possui um caráter endógeno, sendo determinado geneticamente. Halberg apud Withrow (1959), introduziu o termo circadiano (latim circa: em torno de; dies: dia), para caracterizar os ritmos com períodos de 24 horas, os quais são sincronizáveis em ciclos de 24 horas de claro/escuro.

De acordo com Cipolla-Neto et al. (1988), no estudo do ritmo circadiano podem ser destacados dois objetivos principais. O primeiro seria a demonstração de que qualquer variável fisiológica não se mantém estável e constante durante 24 horas, apresentando uma flutuação diária regular, filogeneticamente incorporada e geneticamente determinada, cuja finalidade seria preparar o organismo de uma maneira antecipada para enfrentar modificações ambientais, como a alternância do dia e da noite. O segundo objetivo seria mostrar que as alterações circadianas não se dão apenas no nível da variável observada, mas também e principalmente, na capacidade do organismo em reagir diante de estímulos ambientais e endógenos. Os autores ainda ressaltaram que, dependendo da hora e do dia, os organismos possuem diferenças fisiológicas e comportamentais, podendo responder de maneira diferente aos estímulos.
Na literatura vários autores como DE Coursey (1960), Prosser (1973), Aschoff $(1979,1981)$, Pittendrigh apud Aschoff (1981), Kippert (1985), Cipolla-Neto et al. (1988), Moore \& Rankin (1993), Moore et al. (1998), Moore (2001), destacaram a importância dos conceitos relativos aos ritmos circadianos endógenos e exógenos influenciados por fatores ambientais como as variações diárias de luminosidade (ciclo claro/escuro) e de temperatura.

O objetivo do presente trabalho foi determinar as taxas respiratórias de abelhas forrageiras isoladas de T. a. fiebrigi, $T$. a. angustula e T. spinipes, fazendo uma análise comparativa das respostas fisiológicas desses insetos, para verificar o ritmo circadiano de cada espécie em condições de ciclo claro/escuro ambiental 12:12 (dia/claro e noite/escuro), na primavera.

\section{MATERIAL E MÉTODOS}

As abelhas forrageiras de T. a. fiebrigi, T. a. angustula e $T$. spinipes foram coletadas, com auxílio de um sugador manual, de ninhos capturados na bacia do rio Tibagi, Paraná e mantidos no meliponário da Universidade Estadual de Londrina, Paraná. Foram transportadas para uma câmara climática com temperatura em torno de $25^{\circ} \mathrm{C}$, onde foram realizados os experimentos com respirômetro de Warburg durante a primavera, em temperatura constante de $30^{\circ} \mathrm{C}$ (temperatura média encontrada por Proni \& Hebling (1996) na área de cria de T. a. fiebrigi e T. a. angustula e por ZuCCHI \& SAKAGAMI (1972) na mesma área para T. spinipes), em condições de ciclo claro/escuro ambiental 12:12 (dia/claro e noite/escuro), na primavera.

As leituras do respirômetro foram feitas durante uma hora, com intervalos de três horas, durante 24 horas, sendo que as leituras noturnas foram realizadas com luz vermelha (comprimento de onda não captado pelas abelhas), para evitar estímulos luminosos.

Em todos os experimentos foram utilizados dez frascos contendo uma abelha operária cada e um sem inseto, que funcionou como termobarômetro. No poço central foi colocada uma fita de papel de filtro e $0,04 \mathrm{ml}$ de solução de $\mathrm{KOH}$ a $14 \%$ para absorver o gás carbônico eliminado pela respiração. No bulbo lateral do frasco foi colocado um algodão embebido em $0,3 \mathrm{ml}$ de solução de sacarose a $50 \%$, como fonte de energia, para os insetos confinados individualmente.

Os conjuntos de frascos e manômetros foram levados ao banho, esperando-se 15 minutos com o sistema aberto para o exterior, para fins de termoestabilização do sistema. Após a termoestabilização, os níveis manométricos foram ajustados a $150 \mathrm{~mm}$ e as torneiras fechadas para a tomada das leituras iniciais, de 15 em 15 minutos, durante 60 minutos.

Ao término de cada fase experimental (10:00-11:00, 14:00-15:00, 18:000-19:00, 22:00-23:00, 02:00-03:00, 06:0007:00, 10:00-11:00) as torneiras manométricas eram abertas, permanecendo assim durante os intervalos de três horas, para equilibrar a atmosfera interior dos frascos com a do meio ambiente. No final do experimento ( 24 horas), os frascos foram 
desconectados dos seus respectivos manômetros, as abelhas foram anestesiadas com éter e retiradas dos frascos para determinação da massa corpórea em balança Mettler. Em seguida foi determinado o volume de cada inseto, colocando-se o mesmo em tubo de centrífuga graduado, ao qual se adicionou água através de uma seringa de $1 \mathrm{ml}$. A quantidade de água que ultrapassou a marca de $1 \mathrm{ml}$ do tubo de centrífuga, correspondia ao volume do animal.

De posse desses volumes, calculou-se a constante de cada conjunto (frasco + manômetro), de acordo com Umbreit et al. (1972). Com a constante de cada conjunto, calculou-se o consumo de oxigênio, multiplicando-se a mesma pela soma das variações manométricas (h). Dividindo-se o consumo de oxigênio, de cada fase experimental (uma hora), pela massa corpórea de cada inseto, foram obtidos os valores de $\mathrm{VO}_{2}$ (taxa respiratória) expressos em $\mathrm{ml} \mathrm{de} \mathrm{O}_{2} / \mathrm{mg}$ (vivo)/hora.

\section{RESULTADOS}

As taxas respiratórias médias de 10 operárias forrageiras isoladas de T. a. fiebrigi, T. a. angustula e T. spinipes, na temperatura de $30^{\circ} \mathrm{C}$, durante 24 horas, com ciclo claro/escuro ambiental 12:12 (dia/claro e noite/escuro), na primavera, constam na figura 1.

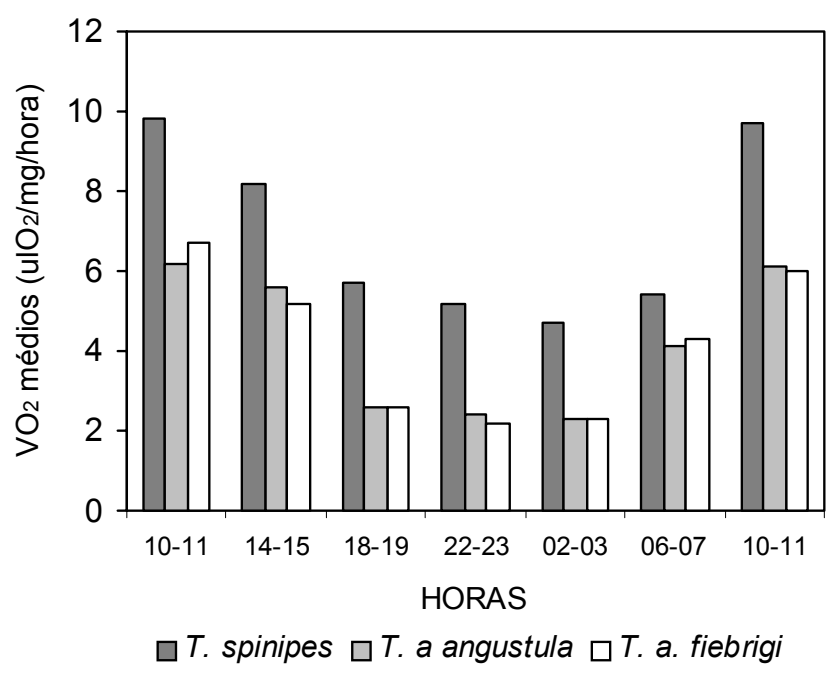

Figura 1. Taxas respiratórias médias $\left(\mathrm{ml} \mathrm{O}_{2} / \mathrm{mg} / \mathrm{hora}\right.$ ) para operárias de duas espécies de abelhas sem ferrão com ciclo ambiental normal 12:12(dia/claro e noite/escuro).

Para análise estatística dos dados referentes às taxas respiratórias médias entre essas espécies e horários: 10-11, 14-15, 18-19, 22-23, 02-03, 06-07 e 10-11 utilizou-se a técnica da análise com dois fatores sem efeito de interação devido à falta das observações individuais que geraram as médias analisadas.
Observada a significância do teste $\mathrm{F}$ da análise de variância procedeu-se ao emprego do teste de TuKey (1953) para comparação das médias duas a duas.

Os testes foram realizados em nível de significância de $5 \%$, logo, valor de $\mathrm{p}<0,05$ foi considerado estatisticamente significativo.

A análise de variância (Tab. I) apresenta a significância do teste $\mathrm{F}$ para os fatores de estudo: espécies ( $\mathrm{p}<0,0001)$ e horário ( $\mathrm{p}<0,0001$ ), ou seja, observou-se efeito significativo entre espécies e horário ao avaliar a taxa respiratória.

Tabela I. Análise de variância com dois fatores: espécie e horário, sem efeito da interação para VO2 médios ( $\mu \mathrm{l} \mathrm{O} 2 / \mathrm{mg} / \mathrm{hora}$ ).

\begin{tabular}{lrrrrr}
\hline C.V. & G.L. & S.Q. & Q.M. & F & $\begin{array}{c}\text { Valor de } \\
\text { p }\end{array}$ \\
\hline Espécie & 2 & 35,8438 & 17,9219 & 76,55 & $<0,0001$ \\
Horário & 6 & 66,1848 & 11,0308 & 47,11 & $<0,0001$ \\
Resíduo & 12 & 2,8095 & 0,2341 & & \\
\hline Total & 30 & 104,8381 & & & \\
\hline
\end{tabular}

Coeficiente de variação $=9,47 \%$.

A tabela II apresenta as médias e o teste de Tukey para comparação das médias duas a duas. Avaliando a taxa respiratória das espécies verificou-se que T. a angustula e T. a fiebrigi não apresentaram diferença significativa, mas estas diferiram da espécie $T$. spinipes, que apresentou médias de taxas respiratórias maiores. Em relação ao horário, para ambas as espécies, verificou-se que não houve diferença significativa entre o inicial, 10-11 e 14-15, e o final 10-11 do dia seguinte, porém estes diferiram dos horários 18-19, 22-23, 02-03 e 06-07. Entre estes últimos observou-se que no intervalo entre as 02-03 as taxas respiratórias médias caíram significativamente diferindo dos horários iniciais anteriores.

\section{DISCUSSÃO E CONCLUSÕES}

De acordo com Cipolla-Neto et al. (1988), no estudo do ritmo circadiano podem ser destacados dois objetivos principais: o primeiro, seria a demonstração de que qualquer variável fisiológica não se mantém estável e constante durante 24 horas, apresentando uma flutuação diária regular, filogeneticamente incorporada e geneticamente determinada, cuja finalidade seria a de preparar o organismo de uma maneira antecipada para enfrentar modificações previamente estabelecidas pelo ambiente, como as alternâncias do dia e da noite; e o segundo objetivo, seria o de mostrar que as alterações no ritmo circadiano não se dão apenas quanto no nível da variável observada, mas também, e principalmente, na capacidade do organismo em reagir diante de estímulos ambientais e endógenos. Os autores ainda observam que, dependendo da hora e do dia, os organismos possuem diferenças 
Tabela II. Média e desvio padrão (DP) da taxa respiratória ( $\mu$ l O2/mg/hora)para operárias de duas espécies de abelhas sem ferrão com ciclo ambiental normal 12:12 (dia/claro e noite/escuro), e teste de Tukey (5\%).

\begin{tabular}{|c|c|c|c|c|c|c|c|c|c|c|}
\hline \multirow{2}{*}{ Espécie } & \multicolumn{7}{|c|}{ Horário } & \multirow{2}{*}{ Média } & \multirow{2}{*}{$\mathrm{DP}$} & \multirow{2}{*}{ Tukey } \\
\hline & $10-11$ & $14-15$ & $18-19$ & $22-23$ & $02-03$ & $06-07$ & $10-11$ & & & \\
\hline Trigona spinipes & 9,80 & 8,20 & 5,70 & 5,20 & 4,70 & 5,40 & 9,70 & 6,96 & 2,21 & A \\
\hline T. a. angustula & 6,20 & 5,60 & 2,60 & 2,40 & 2,30 & 4,10 & 6,10 & 4,19 & 1,78 & B \\
\hline T. a. fiebrigi & 6,70 & 5,20 & 2,60 & 2,20 & 2,30 & 4,30 & 6,00 & 4,19 & 1,86 & B \\
\hline Média & 7,57 & 6,33 & 3,63 & 3,27 & 3,10 & 4,60 & 7,27 & & & \\
\hline DP & 1,95 & 1,63 & 1,79 & 1,68 & 1,39 & 0,70 & 2,11 & & & \\
\hline Tukey ${ }^{2}$ & $\mathrm{a}$ & $\mathrm{a}$ & bc & bc & c & $\mathrm{b}$ & a & & & \\
\hline
\end{tabular}

(1) Teste de Tukey (5\%) para espécies: (= 3,77278 e dms =0,69. Espécies com letras maiúsculas iguais não apresentam diferença estatisticamente significativa; (2) Teste de Tukey (5\%) para horário: $(=4,94690$ e dms =1,382. Horários com letras minúsculas iguais não apresentam diferença estatisticamente significativa. (() valor crítico da amplitude estudentizada; (dms) diferença mínima significativa para o teste de Tukey.

fisiológicas e de comportamento, podendo responder de maneira diferente a um mesmo estímulo, quando aplicado em momentos distintos durante as 24 horas do período.

Segundo Cloudsley-Thompson (1980 apud Cipolla-Neto et al. 1988), os animais podem ser geralmente divididos em diurnos, noturnos ou crepusculares, de acordo com a distribuição de maior atividade durante as 24 horas. Isso não quer dizer que o animal passará todo o dia, toda a noite ou o crepúsculo em atividade contínua, pois existem picos de movimentação característicos para cada espécie animal.

Os resultados obtidos sugerem que a luz desempenhou um papel importante no ritmo circadiano da taxa respiratória, uma vez que as medidas realizadas entre 10:00 e 15:00 e entre 18:00 e 07:00 mostraram, respectivamente, uma elevação e uma queda do consumo de oxigênio, e que realmente está em acordo com observações de campo (saída do ninho para atividade forrageira) para as espécies T. spinipes, T. a. fiebrigi e T. $a$. angustula, sendo que a atividade externa desta última espécie foi estudada por Iwama (1977).

Bellusci \& Marques (2001) estudaram o ritmo circadiano da atividade forrageira de abelhas indígenas sem ferrão Scaptotrigona aff depilis (Moure, 1942), mantendo-as em condições de luminosidade constante e com disponibilidade de alimento. Os resultados mostraram que essa atividade possui um ritmo circadiano endógeno iniciado antes do ciclo natural de claro/escuro e provavelmente influenciado antes de outros ciclos ambientais como temperatura e a disponibilidade de fontes de alimento.

Frisch \& KoENIGER (1994) observaram a sincronização social dos ritmos de atividade de abelhas isoladas individualmente e da colônia de Apis mellifera capensis (Escholtz), constatando que depois de 12 dias de isolamento de abelhas individuais, toda a coincidência entre as fases desses dois ritmos (abelhas individuais e colônia) foi perdida, concluindo-se que os períodos comuns de atividade e descanso resultaram de uma sincronização mútua (social) em contraposição aos ritmos das abelhas individuais.
Moore et al. (1998) estudaram a ontogênese da ritmicidade circadiana em operárias de Apis mellifera (Linnaeus, 1758), demonstrando que esse mecanismo assegurou comportamentos essenciais da colmeia que foram executados ao redor de um ritmo, permitindo que o mesmo seja acionado antes do começo de forragear.

Moore (2001) fez uma revisão de trabalhos para $A$. mellifera mostrando a ocorrência de um ritmo circadiano endógeno, tanto no controle de comportamento de abelhas forrageiras individuais, como para ritmos da colônia inteira.

GiMENEs (2001) observou, em laboratório, a atividade diária de Tetraglossula anthracina (Michener, 1989) em condições de luz e temperatura constantes ( 24 horas) Estas abelhas foram mantidas em caixas e alimentadas. Apresentaram ritmo de atividade e repouso, iniciando suas atividades diárias entre 08.00 e 08.35 e terminando entre 15.00 e 17.00 . Os resultados indicaram a presença de ritmo circadiano endógeno para a atividade diária dessa espécie.

SHIMizu et al. (2001) também observaram, em abelhas forrageiras (isoladas individualmente) de Apis cerana japonica (Radoszkowski, 1887), testadas em condições de luminosidade e escuro constantes durante 24 horas, um ritmo circadiano que controlou a atividade locomotora dessa espécie.

Entretanto, para uma confirmação de que o ritmo apresentado é do tipo exógeno, serão necessários novos experimentos, em condições constantes (durante 24 horas) de claro ou escuro e com inversão de ciclo claro/escuro ambiental 12:12 (dia/escuro e noite/claro), os quais serão realizados futuramente. Deve ser ressaltado, também, que os dados aqui apresentados se referem às abelhas forrageiras estudadas isoladamente, pois como esses insetos são eusociais, outros fatores (além dos ambientais) poderiam influenciar no seu ritmo metabólico, tais como efeito de grupo, presença de cria, diferentes castas, etc.

Os ritmos diários presentes em muitos tipos de comportamentos e em funções fisiológicas e bioquímicas, segundo TAKAHASHI \& ZATZ (1982) podem ser produzidos nos osciladores endógenos que funcionam 24 horas como um relógio interno. 
Em condições naturais esses osciladores são sincronizados para o período normal diário de luz e escuro.

De acordo com PiAnKa (1982), a ecofisiologia trata das relações e funções dos organismos em seus ambientes físicos (luz, temperatura e umidade), de como se adaptam e respondem a eles e de como os exploram. Assim, a ecofisiologia está interessada basicamente nos mecanismos funcionais e comportamentais mediante os quais os organismos agem frente ao meio ambiente, sendo que os mecanismos fisiológicos devem refletir as condições ecológicas. Essas respostas refletem uma variação do ritmo circadiano do consumo de oxigênio havendo, portanto, uma adaptação metabólica modulando com eficiência a variação espacial e temporal do ambiente físico, sendo que permite que os organismos continuem existindo, numa ampla gama de variações ambientais externas, incrementando desta maneira a eficácia biológica.

Outros insetos tiveram o ritmo circadiano da taxa respiratória estudados como por exemplo Banks et al. (1975) que pesquisaram o ritmo circadiano da taxa respiratória de duas espécies de baratas Blaberus craniifer (Burmeister) e Blaberus giganteus (L.) (Dictioptera, Blaberidae), em condições de ciclo normal claro/escuro 12:12 e em escuro e luminosidade constantes (24 horas), sendo que observaram um ritmo circadiano endógeno. Também Chiba et al. (1973) e TAKAHASHI-DEL-Bianco \& Hebling (1992), estudaram a ritmicidade metabólica de insetos Tribolium confusum (Duv.) (Coleoptera) e Camponotus rufipes (Fabricius, 1775) (Hymenoptera, Formicidae), respectivamente, indicando que houve uma flutuação rítmica da taxa respiratória sob condições de ciclo normal claro/escuro 12:12 para as duas espécies. Tweedy \& STEPhen (1971), estudando os efeitos de luz e temperatura no ritmo circadiano do consumo de oxigênio de Megachile rotundata (Fabricius, 1787) (Hymenoptera, Apidae), observaram que o ritmo metabólico mudava de períodos predominantemente curtos (ultradianos) nos estágios imaturos, para um período circadiano estável no estágio adulto.

Uma análise dos dados encontrados na literatura para $A$. mellifera indicou que esses insetos, quando estudados isoladamente, numa faixa de temperatura de 15 a $30^{\circ} \mathrm{C}$, apresentaram um ritmo circadiano no uso de energia caracterizado por dois períodos bem definidos: um período diurno, durante o qual a taxa metabólica é bem acentuada e um período noturno com o metabolismo bem mais baixo (STUSSI 1966, 1968, 1972a, b, STUSSI \& Heusner 1963, Heusner \& Roth 1963, Heusner \& Stussi 1964).

Para meliponíneos Proni et al. (2001) determinaram o ritmo circadiano, com ciclo ambiental normal 12:12 (dia/claro e noite/escuro), do consumo de oxigênio para operárias forrageiras isoladas de Plebeia droryana (Friese, 1900), cujos resultados indicaram um ritmo circadiano bem definido, mostrando uma atividade diurna para esta espécie a $30^{\circ} \mathrm{C}$.

TwEEDY \& STEPHEN (1971) citaram vários autores que reconheceram que, tanto a temperatura como a intensidade luminosa, poderiam modificar as fases de atividade dentro de um período de 24 horas. Entretanto, anteriormente NeL (1967) es- tudou o comportamento de Iridomyrmex detectus (Smith) e verificou que a atividade dessas formigas foi influenciada pela reação à umidade, enquanto que a reação à luz foi menos importante.

Para complementar os dados referentes ao ritmo circadiano das taxas respiratórias de T. a. fiebrigi, T. a. angustula e T. spinipes, também deverão ser realizados estudos sobre a atividade externa diurna, influência da temperatura no metabolismo respiratório de grupos de dois, cinco e 10 insetos, em relação à sazonalidade (verão e inverno), para se determinar um possível efeito de grupo ou não.

\section{REFERÊNCIAS BIBLIOGRÁFICAS}

ABsy, M.L. \& W.E. KerR. 1977. Algumas plantas visitadas para obtenção de pólen por operárias de Melipona seminigra merrillae. Acta Amazônica, Manaus, 7: 309-315.

Absy, M.L.; E.B. Bezerra \& W.E. Kerr. 1980. Plantas nectaríferas utilizadas por duas espécies de Melipona da Amazônia. Acta Amazônica, Manaus, 10: 271-81.

Absy, M.L.; J.M.F. Camargo \& W.E. Kerr. 1984. Espécie de plantas visitadas por meliponinae. (Hymenoptera: Apoidea), para coleta de pólen na região do Amazonas. Revista Brasileira Biologia, Rio de Janeiro, 44 (22): 227-237.

Aschoff, J. 1979. Circadian rhythms: general features and endocrinological aspects, p. 145-198. In: D.T. KRIEGER (Ed.). Comprehensive endocrinology: endocrine rhythms. New York, Raven Press, 488p.

.1981. Biological Rhythms. New York, Plenum Press, IX+645p.

Banks, W.M.; A.S. Bruce. \& H.T. Peart. 1975. The effects of temperature, sex and circadian rhythm on the oxygen consumption in two species of cokroaches. Comparative Biochemistry Physiology, Great Britain, 52 (A): 223-227.

BAWA, K.S. \& O.P. Opler. 1975. Dioesism in tropical forest trees. Evolution, Lawrence, 29: 167-179.

Bellusci, S. \& M.D. Marques. 2001. Circadian activity rhythm of the foragers of a eusocial bee (Scaptotrigona aff depilis, Hymenoptera, Apidae, Meliponinae) outside the nest. Biological Rhythm Research, Liverpool, 32: 117-124.

Bloch, G.; J.P. SulLIVAN \& G.E. Robinson. 2002. Juvenile hormone and circadian locomotor activity in the honey bee Apis mellifera. Journal of Insect Physiology, Urbana, 48 (12): 1123-1131.

Снiba, Y.; L.K. Cutкomp \& F. Halberg. 1973. Circadian oxygen consumption rhythm of the flour beetle, Tribolium confusum. Insect Physiology, University of California, 19: 2163-2172.

Cipolla-Neto, J.; N. Marques \&, L.S. Menna-Barreto. 1988. Introdução ao estudo da cronobiologia. São Paulo, Icone Edusp, XIV+270p.

DE Coursey, P.J. 1960. Daily light sensitivity rhythm in a rodent. Science, Cambridge, 131: 33-35.

Frisch, B. \& N. Koeniger. 1994. Social synchronization of the

Revista Brasileira de Zoologia 21 (4): 987-993, dezembro 2004 
activity rhythms of honey bees within a colony. Behavioral Ecology and Sociobiology, Wurzburg, 35: 91-98.

Gimenes, M. 2001. Preliminary study of activity rhythm of Tetraglossila anthracina (Michener, 1989) (Colletidae, Apoidea) in controlled conditions of laboratory. Revista Brasileira de Zoociências, Juiz de Fora, 3: 44031-460.

HARKER, J.E. 1958. Diurnal rhythms in the animal Kingdom. Review of Biology, Chicago, 33 (1): 1-52.

Heusner, A. \& T. Stussi. 1964. Metabolisme energétique de l'abeille isolée: son role dans la thermorégulation de la ruche. Insects Sociaux, Paris, 11 (3): 239-266.

IWAma, S. 1977. A influência de fatores climáticos na atividade externa de Tetragonisca angustula (Apidae: Meliponinae). Boletim de Zoologia, São Paulo, 2: 189-201.

Jansen, D.H. 1975. Ecology of Plants in the Tropics. London, Ed. Arnold, XIII+641p.

KAPOOR, N.N. 1972. Oxygen consumption of Paragnetina media (Walker): light effect on respiratory rates. Experimentia, Munich, 28: 1311-1312.

KerR, W.E.; R. Cunha; \& J.F. Pisani. 1978. Genética de determinação de sexo. Aplicação de métodos numéricos para agrupar sexos e castas de Melipona quadrifasciata anthidioides Lep. (Apidae). Revista Brasileira de Biologia, Rio de Janeiro, 38: 319-394.

Kerr, W.E.; G.A Carvalho \& V.A. Nascimento. 1996. Abelha uruçu: biologia, manejo e conservação. Belo Horizonte, Ed. Fundação Acangaú, XII+143p.

KIPPERT, F. 1985. Chrono-colloquium on the origin of circadian rhythms. Journal of the Inter-Discipline Cycle Research, Tubingen, 16: 17-84.

Michener, C.D. 1974. The social behaviour of bees. A comparative study. Cambridge, Harvard University, XI+404p.

MOoRe, D. 2001. Honey bee circadian clocks: behavioral control from individual workers to whole-colony rhythms. Journal of Insect Physiology, Johnson City, 47 (8): 843-857.

Moore, D. \& M.A. Rankin. 1993. Light and temperature entrainment of a locomotor rhythm in honey bees. Physiological Entomology, Johnson City, 18 (3): 271-278.

Moore, D.; J.E. Angel; I. M. Cheeseman; S.E. Fahrbach \& G.E. Robinson. 1998. Timekeeping in the honey bee colony: integration of circadian rhythms and division of labor. Behavioral ecology and sociobiology, Johnson City, 43 (3): 147160

Moritz, R.F.A. \& P. Kryger. 1994. Self-organization of circadian rhythms in groups of honey bees (Apis mellifera L.). Behavioral Ecology and Sociobiology, Berlin, 34 (3): 211-215.

NeL, J.I.C. 1967. Reactions of workers of the meat ant Irídomyrmex detectus (Smith) (Dolichonerinae: Hymenoptera) to light and atmospheric humidity. Journal of the Entomological Society of Southern Africa, Hatfield, 30 (1): 111-115.

Nogueira-Neto, P. 1970. A Criação de Abelhas Indígenas Sem Ferrão. São Paulo, Ed. Chácaras e Quintais, X I+365p.

PIANKA, E.R. 1982. Ecología Evolutiva. Barcelona, Ed. Omega
S.A., XI+365p.

Price, P.W. 1975. Insect Ecology. New York, John Wiley \& Sons, $\mathrm{XI}+514 \mathrm{p}$.

Proni, E.A. \& M.J.A. Hebling.1996. Thermoregulation and respiratory metabolism in two brazilian stingless bee subspecies of different climatic distribution, Tetragonisca angustula fiebrigi and T. a. angustula (Hymenoptera: Apidae: Meliponinae), Entomologia Generalis, Stuttgart, 20 (4): 281-289.

Proni, E.A.; M.F. Nogueira \& O.J.D. Macieira. 2001. Ritmo circadiano da taxa respiratória de operárias isoladas de Plebeia droryana (Hymenoptera: Apidae: Meliponinae), durante o inverno e verão. Arquivos de Ciências Veterinárias e Zoologia, Umuarama, 5 (1): 51-58.

Prosser, C.L. 1973. Comparative Animal Physiology. Philadelphia, W.B. Saunders, XIII+966p.

RoubiK, D.W. 1979. Nest and colony characteristics of stingless bees from French Guiana. Journal of the Kansas Entomological Society, Lawrence, 52: 443-470.

Shimizu, I..; Y. Kawai; M. Taniguchi \& S. Aoki. 2001. Circadian rhythm and cDNA cloning of the clock gene period in the honeybee Apis cerana japonica. Zoological Science, Kyoto, 18 (6): 779-789.

STussi, T. 1966. Comparaison à $15^{\circ} \mathrm{C}$ de la consommation d'oxygene de l'abeille isolée et de l'abeille en groupe de 2 et de 10. Centre de la Recherche de la Académie des Sciences, Paris, 263: 1608-1611.

— 1968. Analyse de la variation nyctémérale de la consomation d'oxygène de l'abeille butineuse ísolée place à diferentes temperatures. Annales des Épiphyties, Paris, 19 (1): 145-146.

. 1972 a. Influence de l'abaissement de la pression partielle d'oxygène sur la consommation d'oxygène de l'abeille. Centre de la Recherche de la Académie des Sciences, Paris, 274: 2775-2778.

— 1972 b. Ontogenèse du rythme circadien de la dépense énergétique chez l'abeille. Archives des Sciences Physiologic, Paris, 26: 161-173.

Stussi, T. \& A. Heusner. 1963. Variation nycthémérale de la consommation $\mathrm{d}$ oxygène chez quelques espèces $\mathrm{d}$ insectes. Centre de la Recherche de la Société de la Biologie, Paris, 157: 1509-1512.

TAKAhashy, J.S. \& M. ZATZ. 1982. Regulation of circadian rhythmicity. Science, Cambridge, 217 (17): 1004-1110.

Takahashy-Del-Bianco, M. \& M.J.A. Hebling. 1992. Circadian oscillatory patterns of oxygen uptake in individual workers of the ant Camponotus rufipes. Physiological Entomology, Amsterdam, 17: 377-383.

Tukey, J.W. 1953. The Problem of Multiple Comparisons. New Jersey, Princeton University, IX+189p.

TwEedy, D.G. \& W.P. STEPHEN. 1971. Light and temperature effects on the oxygen consumption rhythms of the leafcutter bee, Megachile rotundata (FABR.). Comparative Biochemistry Physiology, Gainesville, 38 (A): 213-231. 
Umbreit, W.W.; R. H Burris. \& J. F. Stauffer's. 1972. Manometric \& Biochemical Techniques. Minnesota, Burguess Publ., $\mathrm{XII}+256 \mathrm{p}$.

Withrow, R.B. 1959. Photoperiodism and Related Phenomena in Plants and Animals. Washington, American Association for the Advancement of Science, XIII+431p.
Zucchi, R. \& S.F. SAKAGAmi. 1972. Capacidade termo-reguladora em Trigona spinipes e em algumas outras espécies de abelhas sem ferrão (Hymenoptera: Apidae: Meliponinae), p. 301309. In: C. Cruz-Landim; N.J. Hebling,; E. Lello \& C.S. Takahashi (Eds). Livro em Homenagem a Warwik Estevan Kerr?. Rio Claro, Ed. UNESP, III+315p.

Recebido em 04.V.2004; aceito em 19.XI.2004. 